Fizika Nizkikh Temperatur, 1999, v. 25, No 8/9, p. 944-949

\title{
Influence of an orthodeuterium impurity on the thermal conductivity of solid parahydrogen
}

\author{
O. A. Korolyuk, B. Ya. Gorodilov, A. I. Krivchikov, and V. G. Manzhelii \\ B. Verkin Institute for Low Temperature Physics and Engineering, National Academy of Sciences of Ukraine, \\ 47, Lenin Ave., 310164, Kharkov, Ukraine \\ E-mail: korolyuk@ilt.kharkov.ua \\ Received February 23, 1999
}

\begin{abstract}
The thermal conductivity of solid parahydrogen with orthodeuterium impurity at the impurity concentration from 0.01 to $1 \%$ has been studied in the temperature range from 1.8 to $9 \mathrm{~K}$. The nonlinear concentration dependence of the thermal resistivity of the investigated samples is explained by the fact that the intensity of normal three-phonon scattering processes is comparable to that of resistive processes. The analysis of the experimental results has been made in the framework of the Callaway model by using different models for the impurity phonon scattering. It has been found that the intensity of phonon impurity scattering in parahydrogen with orthodeuterium impurity is essentially higher than classical theory predicts and it is close to analogous scattering in solutions of helium isotopes with the equal reduced molar volume given. It is considered that additional impurity scattering of phonons is connected with the change of force constants and lattice distortion in the vicinity of impurity molecules. The quantitative estimation of the mentioned effects has been made.
\end{abstract}

PACS: $63.20 . \mathrm{Mt}, \mathbf{6 6 . 7 0 . + f}, 67.80 . \mathrm{Gb}, 67.80 . \mathrm{Mg}$

\section{Introduction}

The mechanism of the influence of isotopic impurities on the thermal conductivity of quantum crystals should be more complicated than in a case of classical crystals. An isotopic impurity disturbs the zero vibrations of a quantum crystal, which leads to the appearance of lattice distortion and the change of force constants in the vicinity of an isotopic point defect [1-3]. Therefore, when describing phonon scattering by isotopic point defects in quantum crystals, one needs to take into account not only the difference in the masses of the impurity and matrix molecules but also the two other effects mentioned. If molecules of the solution have rotational degrees of freedom, then one has also to take account of scattering by excitations of rotational motion of molecules. Simultaneous action of several mechanisms of phonon scattering impedes the interpretation of results of thermal conductivity studies. To make easier the extraction of contributions of the distortion and the change of force constants to thermal resistivity it is appropriate to study solutions with central interaction, such molecules have no rotational degrees of freedom. To such solutions one can refer ${ }^{3} \mathrm{He}-{ }^{4} \mathrm{He}$ and solid solutions of spin- nuclear modifications of hydrogens, which molecules are in the ground rotational state with $J=0$ (parahydrogen and orthodeuterium). The thermal conductivity of solid solutions ${ }^{3} \mathrm{He}-{ }^{4} \mathrm{He}$ was studied experimentally in [4-9]. Quantum effects in solid hydrogens are less defined than in solid helium isotopes. However, solid hydrogens have higher ratio of molecular weights of isotopes. As we know, the influence of isotopic impurities on thermal conductivity of solid hydrogen has not previously been studied experimentally. In [10] the influence of $\mathrm{Ne}$ impurity on the thermal conductivity of solid parahydrogen was studied. The closeness of the Lennard-Jones potential parameters of hydrogen and neon permits one to consider neon impurities in parahydrogen as quasi-isotopic ones. However, the analysis of data on the thermal conductivity of solid $p-\mathrm{H}_{2}$ with $\mathrm{Ne}$ impurity did not result in finding the effects due to distortion and the change of force constants in the background of a very high mass effect $\left(M_{\mathrm{Ne}} / M_{\mathrm{H}_{2}}=10\right)$.

The given work represents a study of the thermal conductivity of solid parahydrogen with orthodeuterium impurities with the aim of finding and ana- 
lyzing the contribution of distortion and the change of force constants to phonon scattering.

\section{Theoretical models}

The phonon scattering by isotopic impurities was first studied theoretically by Pomeranchuk in 1942 [11]. At present various versions of the Klemens model (review [12]), the resonance scattering model (review [13]), and the Antsygina-Slyusarev model [14] are used to describe phonon scattering by point defects. In the long-wave limit when an impurity molecule and its vicinity vibrate as a unit, all the scattering mechanisms are of Rayleigh character.

The Klemens model is most widely used to describe phonon scattering by point defects. In addition to the mass defect, this model takes a local change of force constants and the field of lattice distortions around an impurity into account. Matrix and impurity atoms vibrate as a unit with the same vibration amplitudes. The rate of phonon relaxation is proportional to the fourth power of frequency and has the form

$$
\tau_{\mathrm{imp}}^{-1}=\frac{c \Gamma V}{4 \pi s^{3}} \omega^{4}
$$

where $c$ is the impurity concentration; $V$ is the molar volume of matrix; $s$ is the sound velocity in the matrix; $\omega$ is the phonon frequency; and $\Gamma$ is a parameter characterizing the intensity of impurity scattering. The parameter $\Gamma$ expressed through the mass defect $\varepsilon=\Delta M / M$, the local change of force constants $\Delta \varphi / \varphi$, and the relative radial distortion $\Delta \delta / \delta$ has the form [15]

$$
\Gamma=\varepsilon^{2}+2(\Delta \varphi / \varphi-6.4 \gamma \Delta \delta / \delta)^{2},
$$

where $\gamma$ is the Grüneisen constant. The Klemens model is used only in the case of long-wave acoustic phonons. This condition is fulfilled for quantum crystals whose melting temperatures are essentially lower than the Debye temperature $\Theta_{D}$. So, $T_{m}=13.81 \mathrm{~K}, \Theta_{D}(T \rightarrow 0)=118.5 \mathrm{~K}$ [16] for solid parahydrogen in equilibrium with vapor.

Another approach is used in the model by Antsygina and Slyusarev (hereafter AS). It is based on the successive solution of kinetic equation for scattering by a heavy impurity in a rigid lattice taking account of local change of force constants. The rate of phonon relaxation is described by the expression

$$
\tau_{\text {imp }}^{-1}=2 c \omega\left[\frac{g_{1}+g_{2}}{2}+g_{1} g_{2} P(\omega)\right]^{2} R_{2}(\omega) \times
$$

$$
\begin{gathered}
\times\left\{\left[1+\frac{g_{1}+g_{2}}{2}-\left(P(\omega)-R_{1}(\omega)\right)-g_{1} g_{2} P(\omega) R_{1}(\omega)\right]^{2}+\right. \\
\left.+\left[\frac{g_{1}+g_{2}}{2}+g_{1} g_{2} P(\omega)\right]^{2} R_{2}^{2}(\omega)\right\}^{-1}
\end{gathered}
$$

where $g_{1}$ and $g_{2}$ are the parameters of the model; the functions $P(\omega), R_{1}(\omega)$ and $R_{2}(\omega)$ are determined by the phonon spectrum of the matrix. The parameters $g_{1}=-\varepsilon /(\varepsilon+1)$, where $\varepsilon$ is the mass defect $\Delta M / M=\left(M_{i}-M\right) / M, M_{i}$ and $M$ are the masses of impurity and matrix molecules, respectively. The local changes of force constants are taken into account by the parameter $g_{2}=$ $=M_{i} s_{i}^{2} / M s^{2}-1$, where $s_{i}$ and $s$ are the sound velocities for pure components. In the Debye approximation the functions $P(\omega), R_{1}(\omega)$ and $R_{2}(\omega)$ have a form

$$
\begin{gathered}
P(\omega)=1-1.5 \omega / \omega_{D}+3\left(\omega / \omega_{D}\right)^{2}- \\
-3\left(\omega / \omega_{D}\right)^{3} \ln \left(1+\omega_{D} / \omega\right), \\
R_{1}(\omega)=-1-1.5 \omega / \omega_{D}-3\left(\omega / \omega_{D}\right)^{2}- \\
-3\left(\omega / \omega_{D}\right)^{3} \ln \left|1-\omega_{D} / \omega\right|, \\
R_{2}(\omega)=3 \pi\left(\omega / \omega_{D}\right)^{3},
\end{gathered}
$$

where $\omega_{D}$ is the Debye frequency.

At $g_{2}=0$ expression (3) transforms into the expression characteristic of resonance phonon scattering by isotopic impurity. According to (3), the frequency dependence of the phonon relaxation rate is rather complicated and its form depends on the relationship between $g_{1}$ and $g_{2}$. In a general case the relaxation rate decreases sharply and tends to zero near the Debye frequency.

In [17] it has been shown that impurity scattering of phonons in solid solution of Ar in parahydrogen is much better described by the AS model than by the models of Klemens and resonance scattering of phonons.

\section{Experimental technique}

The thermal conductivity of solid solutions of orthodeuterium in parahydrogen was measured by the steady-state method. The sample was grown in a stainless steel tube with an inner diameter of $4.5 \mathrm{~mm}$ and length of $38.5 \mathrm{~mm}$. Two carbon resis- 
tance thermometers TRC-2 (VNIIFTRI) were fixed on the external surface of the tube. One of the thermometers served as a gauge for temperature stabilization, the second served for measuring a temperature difference along the sample.

Hydrogen preliminary purified by a palladium filter with the content of chemical impurities not more than $10^{-7}$ and deuterium of chemical purity $99.67 \%$ were used to prepare solutions. Parahydrogen with $0.21 \%$ of orthohydrogen and orthodeuterium with $2 \%$ of paradeuterium were obtained by conversion of liquid hydrogen and deuterium as a result of contact with $\mathrm{Fe}(\mathrm{OH})_{3}$ at $T=20 \mathrm{~K}$. Gaseous mixtures were prepared in a glass vessel at room temperature. An error in the measurement of deuterium concentration in the mixture was less than $15 \%$ of the indication.

The crystals were grown from a gaseous mixture under pressure 50 Torr (somewhat lower than pressure at the triple point of pure parahydrogen) with the rate $0.13 \mathrm{~mm} / \mathrm{min}$. During the growth of the crystals the temperature of bottom of the cell decreased linearly with time and the difference in temperatures between the top and bottom of the cell was kept up equal to $7 \mathrm{~K}$. After crystallization the sample was annealed. The temperature along the sample was equalized over a period of two hours, fixed equal to $12.5 \mathrm{~K}$ and kept constant for two hours as well. Further, when passing from one measurement to another the sample temperature changed so that the maximum temperature difference along the crystal should not exceed $0.3 \mathrm{~K}$. The procedure described above made it possible to obtain good-quality samples and avoid the influence of anisotropy of $h c p$ lattice of parahydrogen on thermal conductivity of samples [18].

\section{Experimental results and discussion}

Thermal conductivity of three samples of $p-\mathrm{H}_{2}$ and five solutions $\left(p-\mathrm{H}_{2}\right)_{1-c}\left(0-\mathrm{D}_{2}\right)_{c}$ at deuterium concentrations (c) $0.01 \% ; 0.05 \% ; 0.1 \% ; 0.5 \% ; 1 \%$ were measured.

Thermal conductivity of pure $p-\mathrm{H}_{2}$ agrees well with the literature data obtained for polycrystals with grain sizes about $1 \mathrm{~mm}$. The data on the thermal conductivity of crystals $p-\mathrm{H}_{2}$ made it possible to conclude that the samples prepared by the technique described above are polycrystals without a selected crystallographic direction.

Figure 1 presents the experimental data obtained in the given work for pure parahydrogen and $p-\mathrm{H}_{2}-\mathrm{o}-\mathrm{D}_{2}$ solutions. The thermal resistivity of the samples depends nonlinearly on the concentration. As shown in [19], the nonlinear concentration de-

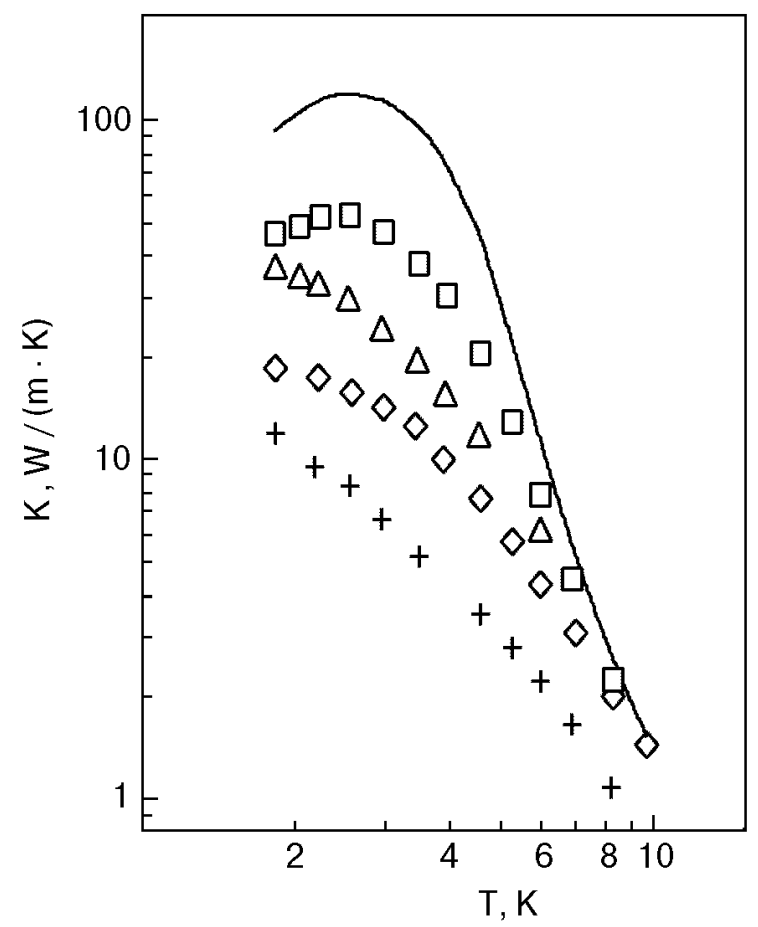

Fig. 1. Temperature dependence of thermal conductivity of $\left(p-\mathrm{H}_{2}\right)_{1-c}\left(o-\mathrm{D}_{2}\right)_{c}$ for different concentrations of $o-\mathrm{D}_{2} \quad c$, \%: 0 (solid line, pure $p-\mathrm{H}_{2}$ ); $0.01(\square) ; 0.05(\Delta) ; 0.1(\diamond) ; 1(+)$.

pendence of thermal resistivity arises due to interference of normal three-phonon scattering processes and impurity scattering. In the temperature range below $4 \mathrm{~K}$ the thermal conductivity curves for different concentrations have various slopes. This shows that in addition to impurity scattering it is also necessary in this temperature range to take into account the scattering on the boundaries of crystalline grains, which intensity changes from sample to sample. The form of temperature dependences of thermal conductivity corresponds to the analogous dependences obtained for ${ }^{3} \mathrm{He}$ with ${ }^{4} \mathrm{He}[9]$.

The analysis of the experimental data was made in the framework of the Callaway model, taking account of normal phonon-phonon scattering processes ( $N$ processes) in the approximation of a Debye phonon spectrum. The thermal conductivity was calculated by the formula

$$
K(T)=G T^{3}\left(I_{1}+I_{2}^{2} / I_{3}\right)
$$

where

$$
I_{1}=\int_{0}^{\Theta / T} \tau_{C}(x, T) f(x) d x ; \quad I_{2}=\int_{0}^{\Theta / T} \frac{\tau_{C}(x, T)}{\tau_{N}(x, T)} f(x) d x ;
$$




$$
\begin{gathered}
I_{3}=\int_{0}^{\Theta / T} \frac{\tau_{C}(x, T)}{\tau_{N}(x, T) \tau_{R}(x, T)} f(x) d x ; \\
G=k_{B}^{2} /\left(2 \pi^{2} \hbar^{3} s\right) ; \quad f(x)=x^{4} \mathrm{e}^{x} /\left(\mathrm{e}^{x}-1\right)^{2} ; \\
x=\hbar \omega / k_{B} T ;
\end{gathered}
$$

$\Theta$ is the Debye temperature; $\tau_{R}(x, T)$ is the relaxation time of resistive scattering processes; $\tau_{N}(x, T)$ is the relaxation time of normal phonon-phonon processes, and $\tau_{C}(x, T)$ is the combined relaxation time:

$$
\tau_{C}^{-1}(x, T)=\tau_{R}^{-1}(x, T)+\tau_{N}^{-1}(x, T) .
$$

In the solutions $\left(p-\mathrm{H}_{2}\right)_{1-c}\left(o-\mathrm{D}_{2}\right)_{c}$ the resistive processes are formed by phonon-phonon processes ( $U$ processes $)\left(\tau_{U}(x, T)\right)$, scattering by the boundaries of crystalline grains $\left(\tau_{B}(x, T)\right)$, scattering by molecules of $o-\mathrm{D}_{2}\left(\tau_{I}(x, T)\right)$ :

$$
\tau_{R}^{-1}(x, T)=\tau_{U}^{-1}(x, T)+\tau_{B}^{-1}(x, T)+\tau_{I}^{-1}(x, T) .
$$

The characteristics of three-phonon $U$ and $N$ processes are determined only by the properties of a crystalline $h c p$ lattice of hydrogen and practically do not depend on the presence of impurity molecules at a small impurity concentration:

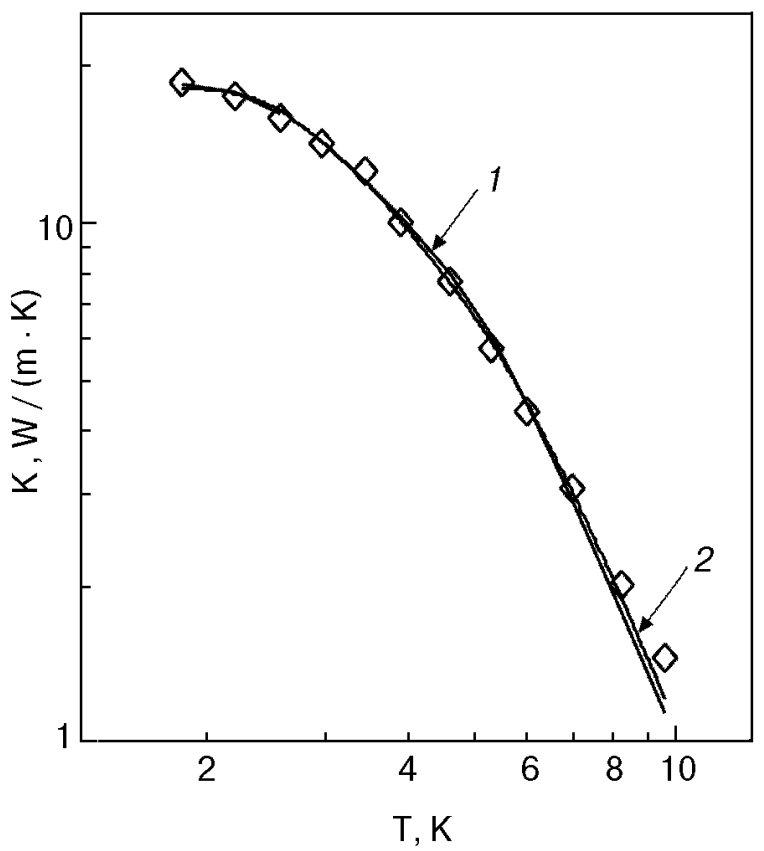

Fig. 2. Temperature dependence of thermal conductivity of solid solution at $c=0.1 \%$ : the experimental data $(\oslash)$, according to the Klemens model (1) and to the AS model (2).

$$
\tau_{U}^{-1}(x, T)=A_{U} x^{2} T^{3} \mathrm{e}^{-E / T}, \tau_{N}^{-1}(x, T)=A_{N} x^{2} T^{5} .
$$

The boundary scattering depends on an average size of crystalline grains $L$ and sound velocity $s$ :

$$
\tau_{B}^{-1}=s / L
$$

The intensity of $N$ processes is taken from the data of [19] $\left(A_{N}=6.7 \cdot 10^{4} \mathrm{~s}^{-1} \cdot \mathrm{K}^{-5}\right)$, and the parameters of $U$ processes are obtained from the data on thermal conductivity of pure parahydrogen ( $\left.A_{U}=5.69 \cdot 10^{7} \mathrm{~s}^{-1} \cdot \mathrm{K}^{-3}, E=36.57 \mathrm{~K}\right)$ [18]. It is worth noting that to describe the thermal conductivity of samples without impurities it was necessary to introduce an additional mechanism corresponding to impurity scattering at the deuterium concentration $c=0.01 \%$. Later it was taken into account when analyzing thermal conductivity of impurity crystals. Note that the natural content of deuterium atoms in hydrogen changes within $(1.39-1.56) \cdot 10^{-4}[20]$.

The analysis of the experimental data was made separately for the impurity scattering models of Klemens and Antsygina-Slyusarev by using dependences (1) or (3), respectively.

To fit the calculated curves to the experimental data we used a mean free path of phonons at boundary scattering $(L)$ and the parameters characterizing the intensity of impurity scattering $\left(\varepsilon_{\text {eff }}, \Gamma\right)$ as the fitting parameters. A parameter $g_{2}$ in the AS model was calculated using the average sound velocities for pure $p-\mathrm{H}_{2}$ and $o-\mathrm{D}_{2}$ obtained from the data on Debye temperatures of these substances. For $p-\mathrm{H}_{2} \quad \Theta=118.5 \mathrm{~K}$ [16] and $s_{\mathrm{H}_{2}}=$ $1342.34 \mathrm{~m} / \mathrm{s}$, for $o-\mathrm{D}_{2} \Theta=111.5 \mathrm{~K} \mathrm{[16]}$ and $s_{D_{2}}=$ $1202.8 \mathrm{~m} / \mathrm{s}$, and $g_{2}=0.606$. For each of the impurity scattering versions the calculated thermal conductivity curves agree satisfactorily with the experimental ones. Figure 2 gives the experimental data for deuterium concentration $c=0.1 \%$ and the calculated dependences of thermal conductivity versus temperature for two models of impurity scattering. The table presents the optimal fitting parameters as a functions of the deuterium concentration.

At deuterium concentration $c \leq 0.1 \%$ the parameters $\Gamma$ and $\varepsilon_{\text {eff }}$ do not depend on concentration within the limits of the experimental error. This means that the deuterium molecules can be considered as independent scattering centers in this concentration range. At $c>0.1 \% \Gamma$ and $\varepsilon_{\text {eff }}$ decrease with increasing concentration. 
Table

Fitting parameters of impurity scattering $\Gamma, \varepsilon_{\text {eff }}$ and averaged mean free path of phonon at boundary scattering $L$, obtained from the experimental data on $K(T)$ for $\left(p-\mathrm{H}_{2}\right)_{1-c}\left(o-\mathrm{D}_{2}\right)_{c}$.

\begin{tabular}{c|c|c|c|c|c}
\hline \hline \multirow{2}{*}{$c, \%$} & \multicolumn{3}{|c|}{ Klemens model } & \multicolumn{2}{c}{ AS model } \\
\cline { 2 - 6 } & $\Gamma$ & $\Delta \delta / \delta$ & $L, \mathrm{~mm}$ & $\varepsilon_{\text {eff }}$ & $L, \mathrm{~mm}$ \\
\hline \hline 0.01 & 1.64 & -0.021 & 0.879 & 2.14 & 1.08 \\
0.05 & 1.64 & -0.021 & 2.50 & 1.98 & 2.68 \\
0.10 & 1.69 & -0.022 & 0.464 & 2.03 & 0.698 \\
0.50 & 1.49 & -0.015 & 0.276 & 1.95 & 0.436 \\
1.00 & 1.19 & -0.001 & 2.18 & 1.72 & 2.68 \\
\hline \hline
\end{tabular}

When treating the results of the thermal conductivity study of solid solutions ${ }^{3} \mathrm{He}-{ }^{4} \mathrm{He}[4-9]$, the authors practically used only the Klemens model. This model makes it possible to compare the increase of intensity of phonon scattering by impurities $\left(\alpha=\Gamma /(\Delta M / M)^{2}\right)$ due to the quantum effects for the cases of solid isotopic solutions of helium and hydrogen. In our opinion, the most accurate thermal conductivity measurements of solutions ${ }^{3} \mathrm{He}-{ }^{4} \mathrm{He}$ were made in works $[8,9]$. According to [9], at molar volume $V=20.56 \mathrm{~cm}^{3} /$ mole and ${ }^{3} \mathrm{He}$ concentration from 0.05 to $0.5 \%$ the values of $\alpha$ changed within 3-3.4. According to Table, for solutions at equilibrium pressure of vapor and low deuterium concentrations $\alpha=1.64 \quad(\alpha=\Gamma$, as $\Delta M / M=1$ ). Thus, at low pressures the quantum isotopic effect in helium is 2 times more, though the mass defect is four times less. It should be noted, however, that the magnitude $\alpha$ characterizing the quantum effect decreases from 3 to 1 , approaching the classical value with decreasing molar volume from 21.56 to $11.5 \mathrm{~cm}^{3} /$ mole. It is interesting to compare $\alpha$ of crystalline hydrogen and helium at equal reduced volumes $V^{*}=V /\left(N \sigma^{3}\right)$, where $\sigma$ is the gas-kinetic diameter of molecules, $N$ is Avogadro's number. If one takes $\sigma=2.62 \AA$ for ${ }^{4} \mathrm{He}$ and $2.96 \AA$ for $\mathrm{H}_{2}$ and $\mathrm{D}_{2}$ [16], then the molar volume of helium $V_{\left({ }^{4} \mathrm{He}\right)}=15.95 \mathrm{~cm}^{3} /$ mole corresponds to the molar volume of hydrogen at equilibrium vapor pressure and helium temperatures ( $V=23.16 \mathrm{~cm}^{3} / \mathrm{mole}$ ). On the basis of the dependence of $\alpha$ on molar volume of solid helium [5,7], we have obtained $\alpha \approx 1.5$ for solid ${ }^{3} \mathrm{He}-{ }^{4} \mathrm{He}$ solutions at $V=15.95 \mathrm{~cm}^{3} /$ mole that is somewhat less then the value $\alpha=1.64$ for solutions $\mathrm{D}_{2}-\mathrm{H}_{2}$ at equal reduced volume.

Let's now estimate the relative change of the force constant $\Delta \varphi / \varphi$ and the relative radial distortion of a lattice $\Delta \delta / \delta$ in the vicinity of the impurity molecule $\mathrm{D}_{2}$ in solid $\mathrm{H}_{2}$. In the framework of
Klemens model [see formula (2)] these effects cannot be determined independently. The magnitude $\Delta \varphi / \varphi$ can be calculated independently from the data on heat capacity of solutions of orthodeuterium in parahydrogen [21]. According to [21], $\Delta \varphi / \varphi=$ $=0.3$. If one uses this value and formula (2), then we obtain the value $\Delta \delta / \delta$ given in Table. The last value can be compared with $\Delta \delta / \delta$ evaluated by the formula

$$
\frac{\Delta \delta}{\delta}=\frac{\left(V_{o-\mathrm{D}_{2}}\right)^{1 / 3}-\left(V_{p-\mathrm{H}_{2}}\right)^{1 / 3}}{\left(V_{p-\mathrm{H}_{2}}\right)^{1 / 3}} .
$$

Using the following values of molar volumes $V_{o-\mathrm{D}_{2}}=$ $=19.9 \mathrm{~cm}^{3} /$ mole and $V_{p-\mathrm{H}_{2}}=23.16 \mathrm{~cm}^{3} /$ mole we obtain $\Delta \delta / \delta=-0.049$. Since both methods of determining the relative radial distortion are rough approximations, the agreement of the values $\Delta \delta / \delta$ obtained by these methods can be considered as satisfactory.

The analysis of the experimental results has shown that the deviations from classical scattering of phonons by an isotopic impurity are observed in crystals of parahydrogen with orthodeuterium impurity. At the same time both the simple and physically rather clear Klemens model and the more consistent AS model describe satisfactorily the impurity contribution to thermal resistivity of a crystal. Both models show the deviation in the behavior of impurity scattering from the classical case. However, the Klemens model (unlike the AS model) permits one to estimate the distortion and the change of force constants in the vicinity of an impurity molecule. The main result of the present work is the discovery and the quantitative estimation of the influence of quantum effects on impurity scattering of phonons in solid hydrogen.

The observed concentration dependences for impurity and boundary scattering give grounds for assuming that experiments with analogous objects made in the lower temperatures range (where the thermal conductivity is mainly determined by a defect structure of crystal) will make it possible to study not only the behavior of isotopic impurity but finer effects as well, related to the influence of impurity on the defect structure of crystal. Note that it is impossible to draw conclusions on the influence of impurities on a defect structure in a sample on the basis of data on the temperature dependence of thermal conductivity in a phonon maximum region. No dependence of boundary scattering parameter on concentration of $\mathrm{D}_{2}$ is observed 
in the solution. To obtain extra information on the influence of macroscopic defects on thermal resistance in $\mathrm{H}_{2}$ it is necessary to widen the region of studying to the side of temperatures lower than $1.7 \mathrm{~K}$.

The authors are indebted to T. N. Antsygina and K. A. Chishko for useful consultations and fruitful discussions of the experimental results.

The authors dedicate this paper to academician B. I. Verkin on the occasion of his 80-th birthday. Owing to his perspicacity and initiative the studies of cryocrystals became possible in our Institute.

1. J. Callaway, Phys. Rev. 122, 787 (1961).

2. P. G. Klemens and A. A. Maradudin, Phys. Rev. 123, 804 (1961).

3. P. G. Klemens, R. De Bruyn Ouboter, and C. Le Pair, Physica 30, 1863 (1964).

4. E. J. Walker and H. A. Fairbank, Phys. Rev. 118, 913 (1960).

5. R. Berman, C.L. Bounds, and S. J. Rogers, Proc. Roy. Soc. (London) A289, 66 (1965).

6. B. Bertman, H. A. Fairbank, Clark W. White, and Michael J. Crooks, Phys. Rev. 142, 74 (1966).

7. R. Berman, C. L. Bounds, C. R. Day, and H. H. Sample, Phys. Lett. A26, 185 (1968)

8. D. T. Lawson and H. A. Fairbank, J. Low Temp. Phys. 11, 363 (1973)

9. A. A. Golub and S. V. Svatko, Fiz. Nizk. Temp. 6, 1112 (1980) [Sov. J. Low Temp. Phys. 6, 540 (1980)].
10. V. G. Manzhelii, B. Ya. Gorodilov, and A. I. Krivchikov, Fiz. Nizk. Temp. 22, 174 (1996) [Low Temp. Phys. 22, 131 (1996)].

11. I. Pomeranchuk, J. Phys. USSR, 6, 237 (1942).

12. R. Berman, Thermal Conductivity in Solids, Clarendon Press, Oxford (1976).

13. C. W. Mccombie and I. Slater, Proc. Phys. Soc. 84, 499 (1964).

14. T. N. Antsygina and V. A. Slyusarev, Fiz. Nizk. Temp. 19, 494 (1993) [Low Temp. Phys. 19, 348 (1993)].

15. G. P. Srivastava, The Physics of Phonons, Adam Hilger, Bristol, Philadelphia and New York (1990).

16. Physics of Cryocrystals, V. G. Manzhelii, Yu. A. Freiman, M. L. Klein, and A. A. Maradudin (eds.) [Part 1, Quantum Molecular Crystals, authored by Vadim G. Manzhelii, Mikhail A. Strzhemechny], AIP Press, Woodbury, New York (1996)

17. B. Ya. Gorodilov, A. I. Krivchikov, V. G. Manzhelii, and N. N. Zholonko, Fiz. Nizk. Temp. 20, 78 (1994) [Low Temp. Phys. 20, 66 (1994)].

18. O. A. Korolyuk, B. Ya. Gorodilov, A. I. Krivchikov, A. S. Pirogov, and V. V. Dudkin, J. Low Temp. Phys. 111, 515 (1998).

19. T. N. Antsygina, B. Ya. Gorodilov, N. N. Zholonko, A. I. Krivchikov, V. G. Manzhelii, and V. A. Slyusarev, Fiz. Nizk. Temp. 18, 417 (1992) [Sov. J. Low Temp. Phys. 18, 283 (1992)].

20. M. P. Malkov, Handbook on Physical-Technical Basis of Cryogenics, Nauka, Moscow (1985).

21. M. I. Bagatskii, I. Ya. Minchina, V. G. Manzhelii, and A. I. Krivchikov, Fiz. Nizk. Temp. 12, 343 (1986) [Sov. J. Low Temp. Phys. 12, 194 (1986)]. 